\title{
KI-Anwendungsfälle zielgerichtet identifizieren
}

Die Identifizierung von wertstiftenden Anwendungsfällen der künstlichen Intelligenz (KI) steht auf der Agenda vieler Unternehmen. Als Beweggründe gelten sowohl das Potenzial der KI, Wettbewerbsvorteile zu erlangen, als auch die Angst, hinter die Konkurrenz zurückzufallen. So scheinen umfangreiche Rechenressourcen, die Verfügbarkeit von Daten, aber auch technologische Durchbrüche beim maschinellen Lernen die Schleusen für die Anwendung von KI in Unternehmen geöffnet zu haben. Die neuen Möglichkeiten, Wettbewerbsvorteile zu erlangen, gehen jedoch mit der Gefahr einher, innovative KI-Anwendungsfälle zu übersehen oder sich auf weniger wertstiftende KI-Anwendungsfälle zu konzentrieren. Daher haben wir eine Methode entwickelt, die Unternehmen dabei unterstützt, wertstiftende $\mathrm{KI}-$ Anwendungsfälle zu identifizieren. Die Praxistauglichkeit und den Nutzen unserer Methode illustrieren wir anhand ihrer Durchführung im Kontext der EnBW AG.

Peter Hofmann, Jan Jöhnk, Dominik Protschky, Philipp Stähle, Nils Urbach und Christoph Buck 
Unternehmen stehen bei der Identifizierung von KI-Anwendungsfällen vor mehreren Herausforderungen: Erstens mangelt es Unternehmen oft an einem umfassenden Verständnis für die mit dem Einsatz von KI einhergehenden Geschäftspotenziale. So kommt es in der Unternehmenspraxis sowohl zu Unter- als auch Überschätzungen. Zweitens müssen KI-Anwendungsfälle für das jeweilige Unternehmen sowohl technisch machbar als auch wirtschaftlich sein und in den individuellen Unternehmenskontext passen. Es ist daher nicht ausreichend, lediglich den KI-Anwendungsfall eines anderen Unternehmens zu kopieren. Drittens stehen Unternehmen vor der Herausforderung, die Lösung zu finden, die am besten zu ihrem individuellen Problem passt. Viertens können KI-Anwendungen, auch unabhängig von einem bestehenden Problem, neue Möglichkeiten zur Gewinnung von Wettbewerbsvorteilen schaffen.

Um diesen Herausforderungen zu begegnen, stellen wir in diesem Beitrag eine Methode vor, mit der KI-Anwendungsfälle strukturiert identifiziert werden können. Beispiele und praktische Einblicke aus der Anwendung der Methode bei der EnBW AG (im Folgenden EnBW genannt), einem deutschen Energieversorgungsunternehmen, ergänzen die theoretische Vorstellung der Methode.

\section{Wozu ist künstliche Intelligenz in der Lage?}

Obwohl das Themenfeld der KI erst kürzlich an Dynamik gewonnen hat, ist das Ziel, Maschinen zu entwickeln, die sich intelligent verhalten, nicht neu. Als KI kann jene Aktivität beschrieben werden, die darauf abzielt, Maschinen intelligent zu machen [1]. Entsprechend bezieht sich die KI auf ein Gebiet der Informatik, das sich mit der Simulation von intelligentem Verhalten befasst [2]. Dieser eher theoretische Definitionsansatz ist jedoch in der Praxis nur bedingt hilfreich. Aufbauend auf der aktuellen Forschung, stellen wir in Tab. 1 daher sieben verständliche und praktisch anwendbare KI-Funktionen vor.

Bestehende KI-Anwendungen folgen ausschließlich der schwachen KIHypothese, die beschreibt, dass sich Maschinen auf eine bestimmte Aufgabe konzentrieren und nicht auf beliebige Aufgaben. Der vorherrschende Ansatz zur Umsetzung von KI beruht dabei auf Methoden des maschinellen Lernens, insbesondere auf künstlichen neuronalen Netzen. Zudem müssen KI-Funktionen nicht an jeder Stelle einer Anwendung verankert sein. So können KI-Funktionen beispielsweise nur an Ein- und Ausgabeschnittstellen genutzt werden (bspw. bei digitalen Assistenten, die natürlichsprachliche Eingaben verstehen), während sich der restliche Teil der Anwendung auf regelbasierte Programmierung stützt.

\section{Eine Methode zur Identifizierung von KI-Anwendungs- fällen}

\section{Ein Überblick der Methode}

Die entwickelte Methode integriert unterschiedliche Aktivitäten in einem iterativen Ablauf (siehe Abb. 1). Zu diesen Aktivitäten zählen sechs Haupt-
Peter Hofmann $n^{1,2}$

Jan Jöhnk $k^{1,2}$

Dominik Protschky ${ }^{2}$

Philipp Stähle ${ }^{3}$

Prof. Dr. Nils Urbach ${ }^{1,2,4}(\bowtie)$

nils.urbach@uni-bayreuth.de

Dr. Christoph Buck

${ }^{1}$ Projektgruppe Wirtschaftsinformatik, Fraunhofer-Institut für Angewandte Informationstechnik FIT, Bayreuth, Deutschland

${ }^{2}$ Wirtschaftsinformatik und Strategisches IT-Management, Universität Bayreuth, Bayreuth, Deutschland

${ }^{3}$ EnBW Energie Baden-Württemberg AG, Stuttgart, Deutschland

${ }^{4}$ Kernkompetenzzentrum Finanz- und Informationsmanagement, Universität Bayreuth, Bayreuth, Deutschland

${ }^{5}$ QUT Business School, Centre for Future Enterprise, Queensland University of Technology, Brisbane, Australien 


\section{Zusammenfassung}

- Einführung verständlicher und praktisch anwendbarer KI-Funktionen

- Beschreibung einer wissenschaftlich fundierten Methode zur Identifizierung von KI-Anwendungsfällen

- Einblicke in die Anwendung der Methode bei EnBW aktivitäten (Eingrenzen, Vorbereiten, Entdecken, Verstehen, Entwerfen und Einführen), welche von einem kontinuierlichen Projekt- und Veränderungsmanagement unterstützt werden. Das Reflektieren und das Zusammenführen der Erkenntnisse jeder Aktivität tragen sodann zur Wissensbasis eines Unternehmens bei.

Bevor sich Unternehmen in Entwicklungszyklen direkt der Identifizierung von KI-Anwendungsfällen widmen können, sollten die Aktivitäten Eingrenzen und Vorbereiten vorausgehen. Das Eingrenzen definiert den Schwerpunkt und die Grenzen der Methodenausführung. Die Vorbereitung zielt darauf ab, Informationen über unternehmensspezifische Kontextfaktoren zu sammeln und zu strukturieren.

Jeder Entwicklungszyklus umfasst die Aktivitäten Entdecken, Verstehen und Entwerfen. Entwicklungszyklen zielen darauf ab, relevante Probleme und Chancen zu identifizieren (Entdecken), ihren Wurzeln auf den Grund zu gehen (Verstehen) und Problemlösungsansätze zu finden (Entwerfen). Darüber hinaus können sogenannte KI-Experimente (zum Beispiel Datenproben, Testen von Annahmen, Proof of Concept) das Verständnis des technologischen Konzepts und der Potenziale der KI fördern. Unternehmen fahren fort, Entwicklungszyklen zu iterieren, bis sie keine neuen KI-Anwendungsfälle mehr finden, diese nicht weiter spezifizieren können oder mit der Menge an KI-Anwendungsfällen zufrieden sind.

Die Durchführung der Methode endet mit der Ableitung der Voraussetzungen für eine erfolgreiche Einführung ausgewählter KI-Anwendungsfälle und der Definition geeigneter Projektaktivitäten.

\section{Die Aktivitäten der Methode im Detail vorgestellt}

\section{Eingrenzen}

Die Aktivität Eingrenzen hilft dem Unternehmen, die Methode zielgerichtet durchzuführen. So bestimmt das Eingrenzen den Bereich der potenziellen KI-Anwendungsfälle und die zur Methodenausführung erforderlichen Ressourcen (zum Beispiel Größe und Zusammensetzung des Projektteams).

\section{Tab. 1 KI-Funktionen}

\begin{tabular}{|c|c|c|}
\hline & Beschreibung & Beispiel \\
\hline Wahrnehmen & Signale aus der realen Welt erfassen und verarbeiten & Reduktion von Bildrauschen \\
\hline Erkennen & Objekte identifizieren oder Konzepte verstehen & Erkennen einer bestimmten Person in einem Bild \\
\hline Erklären & $\begin{array}{l}\text { Ursache-Wirkungs-Paare identifizieren und daraus Schluss- } \\
\text { folgerungen ableiten }\end{array}$ & $\begin{array}{l}\text { Gruppieren von Kundensegmenten, um die zugrunde } \\
\text { liegenden Beziehungen und Strukturen zu erklären }\end{array}$ \\
\hline Entscheiden & zwischen bekannten, diskreten Alternativen auswählen & Entscheiden, ob ein Produkt fehlerhaft ist oder nicht \\
\hline Vorhersagen & zukünftige Ereignisse oder Bedingungen prognostizieren & Vorhersage von Aktienkursen \\
\hline Generieren & etwas Neues produzieren oder schaffen & Generierung von Textantworten für Chatbots \\
\hline Handeln & $\begin{array}{l}\text { Maßnahmen ergreifen, um einen bestimmten Zweck zu errei- } \\
\text { chen oder eine Situation zu bewältigen }\end{array}$ & autonom fahrende Flurförderzeuge \\
\hline
\end{tabular}


Hierbei kann der Anwendungskontext der Methode auf zwei Wegen eingegrenzt werden:

- Priorisierung und Ausschluss bestimmter Bereiche (zum Beispiel Prozesse) oder Technologien (vor allem zu Beginn der Methodenausführung wichtig)

- Priorisierung und Ausschluss bereits identifizierter KI-Anwendungsfälle (erst nach dem ersten Entwicklungszyklus möglich)

Zu diesem Zweck müssen Unternehmen geeignete Bewertungskriterien definieren. $\mathrm{Zu}$ den Bewertungskriterien von EnBW gehörten zum Beispiel die Investitionsrendite, der Zeitraum bis zum Eintritt des Nutzens, die Verfügbarkeit von Ressourcen, die technische Machbarkeit und die Einhaltung der Unternehmenswerte. Zur Bewertung des wirtschaftlichen Potenzials zog EnBW beispielsweise auch neue Geschäftsmodelle in Betracht, die durch die identifizierten KI-Anwendungsfälle ermöglicht werden. Hinsichtlich des Kompromisses zwischen kurz- und langfristigem Nutzen entschied sich EnBW, weder zu kurz (zum Beispiel einfache Ergänzungen zu bestehenden Anwendungen) noch zu weit (zum Beispiel Anwendungen, die Grundlagenforschung erfordern) zu springen. Für die Priorisierung sollten KI-Anwendungsfälle gruppiert werden, die entweder einen vergleichbaren Geschäftseinfluss haben oder technologisch oder kontextuell voneinander abhängig sind. So ermöglichte das Zusammenfassen von KI-Anwendungsfällen im Fall von EnBW, strategische Entscheidungen zu treffen und technologische und kontextbezogene Synergien zu identifizieren.

\section{Vorbereiten}

In den Vorbereitungsphasen vor jedem Entwicklungszyklus werden relevante Informationen über den unternehmensspezifischen Kontext gesammelt

\section{Abb. 1 Ein Überblick über die Methode zur Identifizierung von KI-Anwendungsfällen}

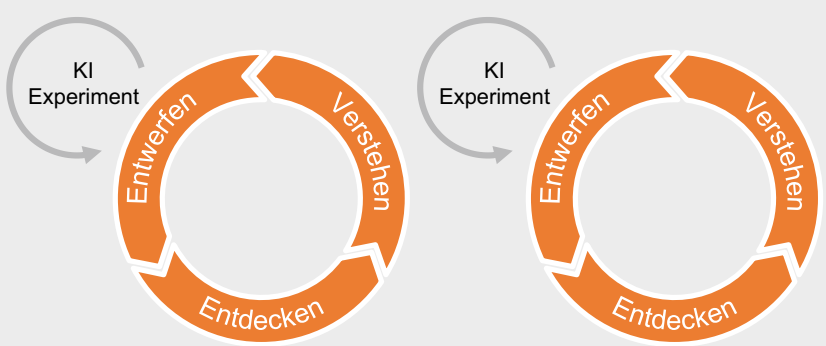

1. Entwicklungszyklus

n. Entwicklungszyklus

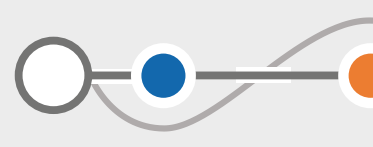

Eingrenzen

\& Vorbereiten

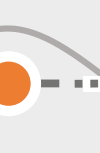

- ".....

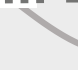

\& Vorbereiten
Veränderungsmanagement

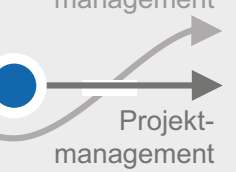

Einführen 


\section{Kernthese 1}

\section{Künstliche Intelligenz kann neue Chancen ermöglichen, nicht nur bestehende Probleme lösen.}

und strukturiert. Da jedes Projekt mit Unwägbarkeiten verbunden ist, sollten die Entwicklungszyklen gründlich vorbereitet werden. Außerdem sollte berücksichtigt werden, dass die Mitglieder des Projektteams typischerweise einen unterschiedlichen Wissenshintergrund haben. Daher sollte das Wissen über Kontextfaktoren über alle Projektmitglieder hinweg konsolidiert werden. Zur Sammlung der relevanten Informationen orientiert sich die Methode am etablierten Technology-Organization-Environment-Rahmenwerk (TOE). Das TOE-Rahmenwerk beschreibt den Einfluss der Faktoren Technologie, Organisation und Unternehmensumwelt auf die Einführung von Technologien [3]. Darüber hinaus sind die Anforderungen der KI im Hinblick auf Daten sowie die Spezifika des gewünschten Anwendungsbereichs zu berücksichtigen.

Technologie. Die Einführung neuer Technologien erfordert von Unternehmen ein Verständnis über ihre bestehende Technologielandschaft und deren Wechselwirkungen mit neuen Technologien. Bei EnBW beispielsweise hängt die Implementierung einiger KI-Anwendungsfälle von der bestehenden IT-Infrastruktur und den Schnittstellen zu Unternehmensanwendungen ab. Darüber hinaus bedarf es eines Verständnisses für die Funktionalität von KI. Im Fall von EnBW hat die Einführung der KI-Funktionen (siehe Tab. 1) das Verständnis für KI wesentlich erhöht. In diesem Zusammenhang sollten Verständnisfragen mit allen relevanten Stakeholdern offen diskutiert werden. Weiterhin muss die bestehende IT-Strategie berücksichtigt und in die Durchführung einbezogen werden. So stellt beispielsweise die IT-Fachbetreuung von EnBW diverse Anforderungen an neue Anwendungen, um unter anderem die Komplexität der IT-Architektur zu bewältigen. Weiterhin ist es wichtig, die bisherigen Erfahrungen und das bestehende Know-how im Unternehmen bezüglich der Anwendung von KI einzubeziehen.

Organisation. In Bezug auf das Unternehmen spielen Aspekte wie Strukturen, Ressourcen und Kultur eine entscheidende Rolle. Es ist wichtig, Technologieentscheidungen an der übergreifenden Unternehmensstrategie auszurichten. So sollten auch die strategischen Ziele und die Konsequenzen der laufenden oder beabsichtigten Umgestaltungen für die Planung und Umsetzung der KI-Projekte einbezogen werden. Darüber hinaus bedarf es der Unterstützung des Topmanagements, einer innovationsfreundlichen Kultur sowie angemessener Ressourcen (zum Beispiel Budget, Mitarbeiter-Knowhow und Verfügbarkeit).

Eine erfolgreiche Anwendung der Methode erfordert ferner, dass der Anwendungsbereich der Methode strukturiert wird. Wenngleich es keine allgemeingültige Richtlinie für die Strukturierung eines Anwendungsbereichs gibt, zählen beispielsweise Produkte, Dienstleistungen, Prozesse oder Probleme zu typischen Kategorien. Beispielsweise stützte sich EnBW auf Prozesse, um seinen Anwendungsbereich aufzugliedern. Insbesondere die technologiebedingten Chancen führten zu neuen Prozessen, die EnBW anfangs noch nicht in Betracht gezogen hatte.

Unternehmensumwelt. Der Umweltkontext umfasst eine Bewertung der Anforderungen der Branche, der Wettbewerber, der Kunden und der Vor- 
schriften. Unternehmen müssen hierbei fördernde und hemmende Umweltfaktoren für die Entwicklung und Implementierung von KI-Anwendungsfällen identifizieren.

Daten. Zudem sind domänenrelevante Daten von großer Bedeutung, da maschinelle Lernmodelle nur dann aussagekräftige Ergebnisse erzeugen können, wenn die benötigten Daten qualitativ und quantitativ für die Anwendung zur Verfügung stehen. Beispielsweise erfordert das Training von Algorithmen für maschinelles Lernen große Datenmengen, um eine angemessene Ausgabequalität zu erreichen. Unternehmen müssen diese Aspekte sorgfältig berücksichtigen und die verfügbaren Daten bewerten (zum Beispiel Aktualität, Datenumfang, Datenqualität, Relevanz, Zugänglichkeit). Die Bewertung beinhaltet auch eine Diskussion der relativen Ressourcenvorteile im Vergleich zu den Konkurrenten.

Anwendungsbereich. Zunächst müssen Unternehmen relevante Informationen des Anwendungsbereichs sammeln, um ein gemeinsames Verständnis zu schaffen (zum Beispiel durch gemeinsame Teams mit KI- und Domänenexperten). Bei EnBW analysierte das Projektteam die Anzahl der Prozessinstanzen, die Prozesskomplexität, die Verantwortlichkeiten, die Werttreiber und die aktuelle Werkzeugunterstützung.

\section{Entwicklungszyklen}

Die Entwicklungszyklen bestehen aus drei Aktivitäten (Entdecken, Verstehen und Entwerfen), die darauf abzielen, KI-Anwendungsfälle für das eigene Unternehmen zu schaffen.

Entdecken. Im Rahmen der Aktivität Entdecken können Unternehmen Handlungsfelder aus zwei Perspektiven identifizieren:

- Domänenperspektive: Aus dieser Perspektive werden Handlungsfelder problemorientiert identifiziert, indem bestehende oder noch unentdeckte Probleme in der Anwendungsdomäne ermittelt und transparent gemacht werden.

- Technologieperspektive: Aus der Technologieperspektive werden Handlungsfelder chancenorientiert identifiziert. So werden (neue) KI-Anwendungen untersucht, um technologiebedingte Chancen in der Domäne zu erkennen.

In der Domänenperspektive gibt es mehrere Ansätze, um Unternehmen bei der Identifizierung von Handlungsfeldern zu unterstützen. Dabei ist zu empfehlen, stets einen stakeholderorientierten Ansatz zu verfolgen. So führte EnBW beispielsweise einen Value-Proposition-Canvas-Workshop mit verschiedenen Stakeholdern durch, um das Kundensegment und die resultierenden Anforderungen zu definieren [4]. Dies ermöglichte es EnBW, die Probleme des Anwendungsbereichs aus Kundenperspektive zu identifizieren.

Aus der Technologieperspektive kann ein Unternehmen eine umfassende Marktanalyse durchführen, um KI-Anwendungen zu identifizieren und Möglichkeiten für den Einsatz für das eigene Unternehmen abzuleiten. Aufgrund des begrenzten Marktes für KI-Anwendungen in bestimmten Berei-

\section{Kernthese 2}

Die Identifizierung von KI-Anwendungsfällen ist mehr als eine technische Herausforderung. 
chen können auch Produkte oder Dienstleistungen außerhalb der eigenen Domäne als Inspiration dienen.

Verstehen. Unternehmen sollten die auf diese Weise identifizierten Handlungsfelder grundlegend verstehen. Hierbei ist eine Ursachenanalyse zu empfehlen, um die Wurzel eines Problems oder einer Chance zu verstehen, nicht nur die Symptome. Dies wird anhand eines Beispiels kurz erläutert: Hohe Kosten für Maschinenausfälle weisen zum Beispiel auf eine unzureichende Wartung hin. Allerdings ist eine häufigere Wartung von Maschinen ineffizient und führt somit zu höheren Kosten. Eine effizientere Wartung von Maschinen erfordert, den Maschinenzustand vorhersagen zu können. Die Ursache des Problems ist ein mangelndes Verständnis der Ausfallursachen (zum Beispiel durch das Sammeln detaillierter Fehlerinformationen, um Wartungsentscheidungen zu leiten). Ebenso könnte eine Chance darin bestehen, den Maschinenbetrieb zu verbessern, um Ausfälle zu verhindern (zum Beispiel durch die Sammlung von Maschinendaten für eine vorsorgliche Maschinenwartung).

Entwerfen. Um konkrete KI-Anwendungsfälle zu entwerfen, müssen Unternehmen Probleme (und Chancen) mit Lösungen abgleichen. Hierzu empfiehlt sich der Einsatz einer Problemlösungsmatrix, in welcher die gesammelten Informationen zusammengefasst werden (siehe Abb. 2). Während die Spalten die Domänenstruktur darstellen (hier im Beispiel anhand der Prozesse), geben die Zeilen die KI-Funktionen an. Das Ziel der Aktivität Entwerfen ist es, innerhalb einer Zelle der Matrix sowohl domänenspezifische Probleme als auch Lösungen zusammenzubringen. Jede Übereinstimmung kann sodann als Anwendungsfall extrahiert werden. Unternehmen sollten die resultierenden KI-Anwendungsfälle mit den Kontextfaktoren vergleichen, Abhängigkeiten zwischen ihnen identifizieren und ihren Mehrwert

\section{Abb. 2 Eine beispielhafte Problemlösungsmatrix}

\begin{tabular}{|c|c|c|c|c|c|}
\hline \multirow[b]{3}{*}{ Wahrnehmen } & \multicolumn{5}{|c|}{ Domänenstruktur } \\
\hline & \multicolumn{2}{|c|}{ Prozess A } & \multicolumn{2}{|c|}{ Prozess B } & \multirow{2}{*}{$\begin{array}{l}\cdots \\
\ldots\end{array}$} \\
\hline & Lösun & Problem & Lösung & Problem & \\
\hline Erkennen & Lösun & Problem & Lösung & Problem & ... \\
\hline Erklären & Lösun & Problem & Lösung & Problem & $\cdots$ \\
\hline Entscheiden & Lösun & Problem & Lösung & Problem & $\cdots$ \\
\hline Vorhersagen & Lösun & Problem & Lösung & Problem & ... \\
\hline Generieren & Lösun & Problem & Lösung & Problem & .. \\
\hline Handeln & Lösun & Problem & Lösung & Problem & $\cdots$ \\
\hline
\end{tabular}


bewerten. Sobald eine Sammlung von KI-Anwendungsfällen vorliegt, kann ein ergebnisorientiertes Eingrenzen durchgeführt werden.

Sollte keine Übereinstimmung zwischen technischer Lösung und einem bestehenden Problem gefunden werden, können zwei unterschiedliche Ansätze zur Lösungsfindung herangezogen werden:

- Problemorientierter Ansatz: Wenn es ein Problem gibt, aber keine direkt ersichtliche Lösung - In diesem Fall kann sich das Unternehmen auf eine Spalte der Matrix konzentrieren, um sich mittels Techniken der Technologieauswahl oder des erfinderischen Problemlösens neue KI-Lösungen für ein Domänenproblem zu erarbeiten.

- Lösungsorientierter Ansatz: Wenn es eine Lösung, aber kein Problem in der Domäne gibt - In diesem Fall kann sich das Unternehmen auf eine Zeile der Matrix konzentrieren. Um diesem Ansatz zu folgen, kann beispielsweise geprüft werden, ob ein Domänenproblem übersehen wurde, dessen Lösung mittels KI neues Geschäftspotenzial birgt (d. h. ein unentdecktes Problem).

\section{Einführen}

In der Aktivität Einführen realisieren Unternehmen die theoretischen Überlegungen in der Praxis und schaffen so die Voraussetzungen für eine erfolgreiche Einführung des KI-Anwendungsfalls. Hierbei sollte zunächst kritisch evaluiert werden, ob andere Technologien geeigneter oder kostengünstiger sind. Um die Einführung eines KI-Anwendungsfalles zu planen, sollte ein Unternehmen die Eckpfeiler der Einführung klären:

- Make or buy: Unternehmen müssen entscheiden, ob die KI-Anwendung intern entwickelt, ausgelagert oder eine bestehende Lösung von einem externen Anbieter gekauft werden soll.

- Reifegrad: Unternehmen müssen den gewünschten Reifegrad der Anwendung bestimmen (zum Beispiel Proof of Concept, minimal realisierbares Produkt, produktionsreife Anwendung).

Kontextfaktoren. Zuletzt sollte der KI-Anwendungsfall vor dem Hintergrund der Kontextfaktoren betrachtet werden, um KI-Anwendungsfälle in das Unternehmen zu integrieren und die Zweckmäßigkeit des KI-Anwendungsfalles zu fördern. Im Hinblick auf den Technologiekontext sollte beispielsweise bedacht werden, wie die KI-Anwendung in die aktuelle IT-Infrastruktur integriert werden kann. Im organisatorischen Kontext sollte das Unternehmen unter anderem auf die Bedenken der Mitarbeiter eingehen und bewerten, ob geeignete Mitarbeiter (zum Beispiel Data Scientists, Machine Learning Engineers, Business Analysten) zur Verfügung stehen. Als Beispiel für den Kontext der Unternehmensumwelt sollte das Unternehmen den Datenschutz und ethische Fragen sorgfältig prüfen. Ebenso sollten datenbezogene Aspekte berücksichtigt werden; wenn zum Beispiel die erforderlichen Daten noch nicht verfügbar sind, muss die Datensammlung geplant werden. In Bezug auf den Anwendungsbereich sollten Unternehmen beispielsweise entscheiden, ob und wie bestehende Prozesse geändert werden sollen, bevor die KI-Anwendung eingeführt wird.

\section{Kernthese 3}

Die Identifizierung von KI-Anwendungsfällen betrifft das gesamte Unternehmen. 


\section{Handlungsempfehlungen}

- KI-Anwendungsfälle strukturiert identifizieren

- KI-Anwendungsfälle in den Unternehmenskontext setzen

- Über den Tellerrand des eigenen Projektteams und der aktuellen KIKenntnisse schauen
Nach diesen Überlegungen kann der KI-Anwendungsfall an ein Projektteam übergeben werden, das die Einführung vorantreibt.

\section{Projekt- und Veränderungsmanagement}

Neben den bisher vorgestellten Hauptaktivitäten besteht die Notwendigkeit, die Methodenausführung im Rahmen des Projektmanagements zu koordinieren. Neben üblichen Fragestellungen des Projektmanagements sollte im Rahmen der vorgestellten Methode insbesondere beachtet werden, dass alle Projektmitglieder mit dem methodischen Ansatz vertraut und bezüglich der angestrebten Eingrenzung des Anwendungsbereichs abgestimmt sind. So sollten unterschiedliche berufliche Hintergründe der Projektteammitglieder berücksichtigt werden. Der Fall EnBW bestätigt, dass diese unterschiedlichen beruflichen Hintergründe die Zusammenarbeit und Kreativität fördern, aber auch die Bedeutung des Wissensaustauschs erhöhen.

Die Entwicklung von Anwendungsfällen und die spätere Implementierung neuer Technologien gehen stets mit Veränderungen einher. Daher sollte ein Unternehmen auch das Identifizieren von KI-Anwendungsfällen mit Veränderungsmanagement begleiten. So sollte das Ziel des Veränderungsmanagements sein, das Bewusstsein und die Wertschätzung für das Projekt und die Motivation der Beteiligten zur Teilnahme am Projekt zu erhöhen. Zur Steigerung der Wertschätzung für das Projekt sollte großer Wert auf eine angemessene Kommunikation gelegt werden. Es ist dabei wichtig sicherzustellen, dass die Mitarbeiter des Unternehmens stets transparente, verständliche und greifbare Informationen erhalten. Hier wird empfohlen, nicht nur KI-Experten, sondern beispielsweise auch Domänenexperten in die Methodenausführung einzubeziehen. Bei EnBW sprach das Projektteam häufig mit Stakeholdern und stellte unter anderem seine Ergebnisse offen auf gedruckten Postern vor. Die Diskussionen reichten von formalen Statusberichtterminen über kreative Workshops bis hin zu informellen Gesprächen bei einem Kaffee.

\section{Zusammenfassung}

Die im Beitrag beschriebene Methode wurde wissenschaftlich fundiert hergeleitet und kann zur Unterstützung bei der Identifizierung von KI-Anwendungsfällen genutzt werden. Dabei stellt die entwickelte Methode keinen isolierten, sondern einen integrierten Ansatz dar, den Unternehmen in der Praxis direkt anwenden können. Insbesondere werden die technische Machbarkeit von KI-Anwendungsfällen, ihre Einbettung in den Unternehmenskontext und ihr wirtschaftlicher Wert berücksichtigt. Darüber hinaus können Unternehmen die Ausgestaltung der Methode an ihren jeweiligen individuellen Bedarf anpassen. Durch die Anzahl der ausgeführten Entwicklungszyklen und die Eingrenzung des Domänen- und Technologieumfangs kann ein Unternehmen den Aufwand für die Ausführung der Methode und damit den Reifegrad ihrer Ergebnisse flexibel anpassen. 
Funding. Open Access funding provided by Projekt DEAL.

Open Access Dieser Artikel wird unter der Creative Commons Namensnennung 4.0 International Lizenz veröffentlicht, welche die Nutzung, Vervielfältigung, Bearbeitung, Verbreitung und Wiedergabe in jeglichem Medium und Format erlaubt, sofern Sie den/die ursprünglichen Autor(en) und die Quelle ordnungsgemäß nennen, einen Link zur Creative Commons Lizenz beifügen und angeben, ob Änderungen vorgenommen wurden.

Die in diesem Artikel enthaltenen Bilder und sonstiges Drittmaterial unterliegen ebenfalls der genannten Creative Commons Lizenz, sofern sich aus der Abbildungslegende nichts anderes ergibt. Sofern das betreffende Material nicht unter der genannten Creative Commons Lizenz steht und die betreffende Handlung nicht nach gesetzlichen Vorschriften erlaubt ist, ist für die oben aufgeführten Weiterverwendungen des Materials die Einwilligung des jeweiligen Rechteinhabers einzuholen.

Weitere Details zur Lizenz entnehmen Sie bitte der Lizenzinformation auf http://creativecommons.org/licenses/by/4.0/deed.de

\section{Literatur}

[1] Nilsson, N. J. (2010). The quest for artificial intelligence: a history of ideas and achievements. Cambridge: Cambridge University Press.

[2] Russell, S., \& Norvig, P. (2016). Artificial intelligence. A modern approach

(3. Aufl.). Edinburgh: Pearson Education.

[3] Baker, J. (2012). The technology-organization-environment framework. In Y. K. Dwivedi, M. R. Wade \& S. L. Schneberger (Hrsg.), Information systems theory. New York: Springer.

[4] Osterwalder, A., Pigneur, Y., Bernarda, G., Smith, A., \& Papadakos, P. (2014). Value proposition design. How to create products and services customers. Hoboken, NJ: Wiley. 